\title{
VARIA
}

\section{UNA CARTA INÉDITA DE EL GRECO AL CARDENAL ALESSANDRO FARNESIO}

Uno de los períodos más oscuros dentro de la carrera italiana de El Greco es el de su estancia en la ciudad de Roma y, concretamente, su actividad al servicio del cardenal Alessandro Farnesio. Apenas llegado de Venecia, es presentado al purpurado en la carta de G. Clovio del 16 de noviembre de 1570. Por su excelencia en el arte de la pintura, le suplica que le aloje en el Palacio Farnesio, a lo que el purpurado accederá. Es desde este momento hasta julio de 1572 cuando se debe fijar la protección farnesiana al pintor.

De la carta que damos a conocer ${ }^{1}$ y que escribe a su patrón, a la sazón en Caprarola, se deduce que una persona relacionada con Farnesio, posiblemente el miniaturista, se puso encontacto con él para que entrase a su servicio. Tras una calculada introducción, redactada con una manifiesta falsa modestia, sale a relucir el talante altivo y consciente del valor de su arte que le caracterizará. Así, El Greco pide explicaciones a Farnesio del trato recibido. En las siguientes líneas es donde se percibe que no debía de ser del todo ignorante de las acusaciones que se cernían sobre él, ya que las califica de falsas y se declara dispuesto a subsanar sus faltas al servicio del cardenal, a quien se compromete servir el tiempo que le resta de vida. A pesar del propósito de enmienda del pintor, parece que éste debió de cometer una falta grave que desencadenó la reacción del cardenal ordenando a su mayordomo que le despidiese y que esta decisión era inamovible.

Esta ruptura brusca, que vendría a avalar las noticias que aporta Mancini sobre la actividad de El Greco en Roma, explicaría la siguiente noticia que conocemos sobre El Greco en Roma, que es su ingreso en la Academia de S. Lucas como pintor independiente el 18 de septiembre de 1572. Aunque se ha interpretado este dato como que se sentía lo bastante maduro en su arte y con una clientela suficiente como para prescindir de la tutela de Farnesio, la realidad era muy diferente.

Otro punto que no se puede pasar por alto en este breve análisis es la supuesta actividad del pintor como fresquista en la villa de Caprarola que queda definitivamente aclarado. La mención que hace del pintor Ludovico Tedesco en la carta que escribe al cardenal el 18 de julio, se refiere a su despido y no a que trabajase en la villa a las órdenes de Bertoia, ni a que actuase como mensajero entre el cardenal y su mayordomo.

\footnotetext{
1 Un estudio más profundo de esta carta y de la estancia del pintor al servicio de la familia Farnesio, enriquecido con otras noticias documentales, se publicará próximamente en la revista Aurea Parma.
} 
* Carta de Domenico Teotocopuli al cardenal Alessandro Farnesio (Caprarola), Roma, 6 de junio de 1572 . Archivio di Stato di Parma, Carteggio Farnesiano, Estero, Roma, busta 367, s.f.

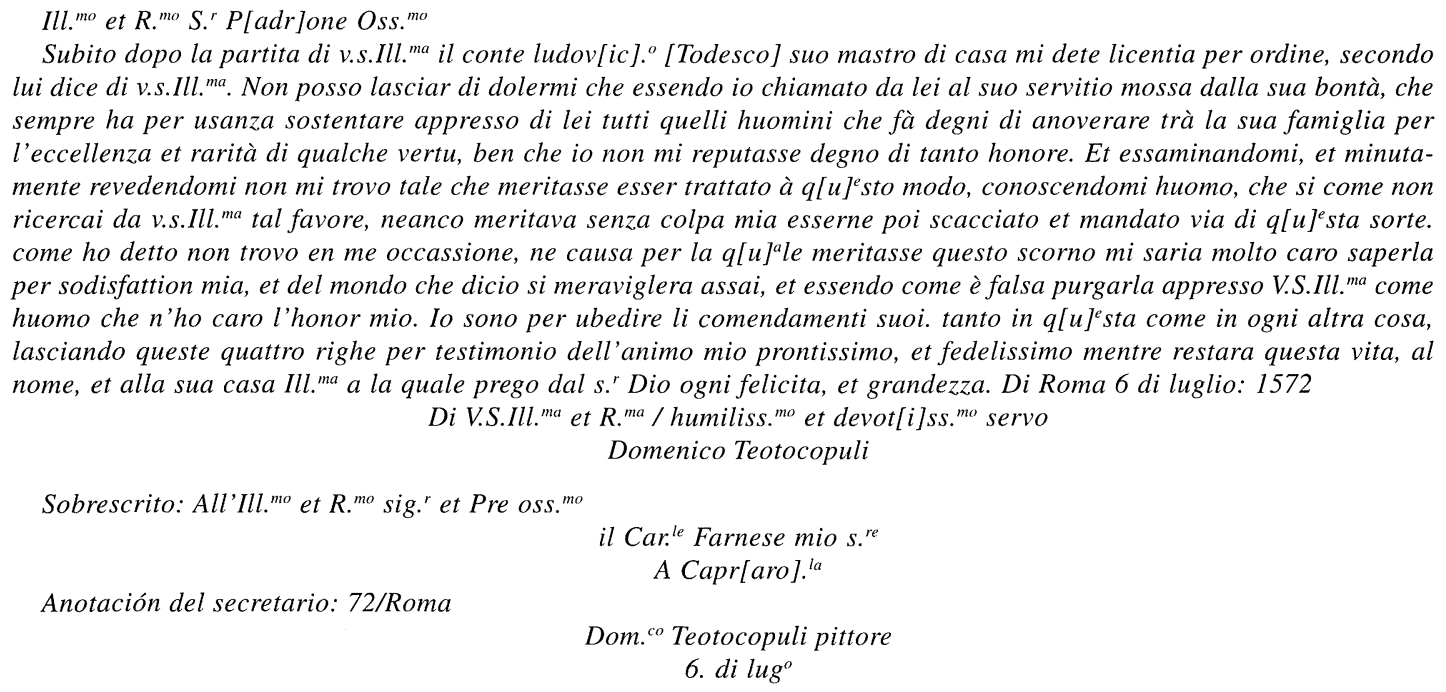

Subito dopo la partita di v.s.Ill. ${ }^{\text {ma }}$ il conte ludov[ic]. ${ }^{\circ}$ [Todesco] suo mastro di casa mi dete licentia per ordine, secondo lui dice di v.s.Ill. ${ }^{m a}$. Non posso lasciar di dolermi che essendo io chiamato da lei al suo servitio mossa dalla sua bontà, che sempre ha per usanza sostentare appresso di lei tutti quelli huomini che fà degni di anoverare trà la sua famiglia per l'eccellenza et rarità di qualche vertu, ben che io non mi reputasse degno di tanto honore. Et essaminandomi, et minutamente revedendomi non mi trovo tale che meritasse esser trattato à q[u] $]^{e}$ sto modo, conoscendomi huomo, che si come non ricercai da v.s.Ill. ${ }^{m a}$ tal favore, neanco meritava senza colpa mia esserne poi scacciato et mandato via di q[u $]^{e}$ sta sorte. come ho detto non trovo en me occassione, ne causa per la q[u] le meritasse questo scorno mi saria molto caro saperla per sodisfattion mia, et del mondo che dicio si meraviglera assai, et essendo come è falsa purgarla appresso V.S.Ill. ${ }^{\text {ma }}$ come huomo che n'ho caro l'honor mio. Io sono per ubedire li comendamenti suoi. tanto in q[u] sta come in ogni altra cosa, lasciando queste quattro righe per testimonio dell'animo mio prontissimo, et fedelissimo mentre restara questa vita, al nome, et alla sua casa Ill. ma a la quale prego dal s. ${ }^{r}$ Dio ogni felicita, et grandezza. Di Roma 6 di luglio: 1572 Di V.S.Ill. ${ }^{\text {ma }}$ et R. ${ }^{\text {ma }} /$ humiliss. ${ }^{\text {mo }}$ et devot [i]ss. ${ }^{\text {mo }}$ servo Domenico Teotocopuli

Sobrescrito: All'Ill. ${ }^{m o}$ et R. ${ }^{m o}$ sig. ${ }^{r}$ et Pre oss. ${ }^{m o}$ il Car. ${ }^{l e}$ Farnese mio s. ${ }^{r e}$ A Capr[aro]. ${ }^{\text {ta }}$

Anotación del secretario: 72/Roma

Dom. ${ }^{\text {co }}$ Teotocopuli pittore 6. di $\mathrm{lug}^{\circ}$

Almudena Pérez de Tudela Becaria de la Universidad Complutense

\section{EL LIENZO DE VERTUMNO Y POMONA DE RUBENS DEL CUARTO BAJO DE VERANO DEL ALCÁZAR DE MADRID}

Vertumno y Pomona, fijados en el primer plano del lienzo, llenan el espacio con la corpórea monumentalidad de sus formas y volúmenes. Sirve de fondo al encuentro de los dioses un frondoso paisaje, abierto a un cielo azul con nubes apacibles, en un limitado espacio a la derecha del espectador. Desde aquí se filtra la luz de la mañana. La tierra fértil, húmeda y frondosa, exuda la savia vital y generosa de los bosques, las plantas, las hortalizas y las frutas, cargados de igual compromiso iconológico que los protagonistas de la historia que narra Rubens (lienzo $116 \times 186,5 \mathrm{~cm})$ (figs. 1-2).

Importa primeramente precisar la identificación propuesta, su valoración técnica y estética, y la documentación histórica que prueba su vinculación al coleccionismo español y extranjero en el curso de este siglo, sin precisiones críticas favorables a su autoría, salvo en mi tesis doctoral donde figura entre las obras posibles de Rubens ', asociándola más tarde al lienzo de Vertumno y Pomona perdido tras el incendio del Alcázar de Madrid ${ }^{2}$ de 1734, donde el número de Rubenes desaparecidos fue considerable ${ }^{3}$.

Sólo una referencia a este lienzo de Vertumno y Pomona encuentro en la monografía de H. Lahrkamp sobre Jan Bockhorst, excluyendo una atribución, certificada por Sturla J. Gudlaugsson, a este discípulo de Rubens, en colección privada de La Haya, en $1982{ }^{4}$. .

\footnotetext{
1 M. Díaz Padrón, La pintura flamenca del siglo xvir en España, (ms.), Madrid, 1976, III, p. 960.

2 Idem, El siglo de Rubens en el Museo del Prado, Barcelona, 1995, I, pp. 24, 49.

${ }^{3}$ A juzgar por los inventarios del Alcázar de 1686 y 1700 debieron perderse cerca de unas cuarenta pinturas de Rubens, sin contar las copias.

${ }^{4}$ H. Lahrkamp, «Der 'Lange Jan', Leben und Werk des Barockmalers Johann Bockhorts», Munster, Westfalen hefte fur Geshichte kunst, I, 1982, p. 173, A 16.
} 